\title{
Prospecção Fitoquímica e Análise da Atividade Antimicrobiana e Moduladora in Vitro do Extrato Hidroalcoólico das Folhas de Senna spectabilis
}

\section{Phytochemical Prospection and Analysis of the Antimicrobial and in Vitro Modulator Activity of the Hydroalcoolic Extract of the Leaves of Senna spectabilis}

\author{
Jessyka Alencar Ferreira*a; Dárcio Luiz de Sousa Júnior ${ }^{\mathrm{b}}$; Ana Emília Formiga Marquesª Rafael de Carvalho Mendes ${ }^{\mathrm{a}}$ \\ ${ }^{a}$ Faculdade de Medicina Estácio de Juazeiro do Norte. CE, Brasil. \\ bUniversidade Regional do Cariri, Programa de Pós-Graduação Stricto Sensu em Química Biológica. CE, Brasil. \\ *E-mail: jessykaalencar91@hotmail.com
}

\begin{abstract}
Resumo
O objetivo do presente estudo foi identificar a composição química da Senna spectabilis, averiguar o potencial antimicrobiano in vitro e analisar seu efeito modulador da atividade antibiótica. Os ensaios fitoquímicos foram realizados seguindo o método proposto por Matos (1997), baseando-se na observação visual de alteração de cor ou formação de precipitado após a adição dos reagentes específicos. Os testes de Concentração Inibitória Mínima (CIM) e modulação foram determinados pelo ensaio de microdiluição em caldo, seguindo as recomendações do Clinical and Laboratory Standards Institute (CLSI). As cepas utilizadas foram Saphylococcus aureus, Escherichia coli, Pseudomonas aeruginosa e Salmonella entérica. Após essas análises se verificou a presença significativa dos metabólitos secundários taninos condensados, alcaloides, esteroides e saponinas. Nos resultados referentes à avaliação da ação antimicrobiana do extrato de S. spectabilis contra as cepas testadas se observou que houve crescimento bacteriano em todas as concentrações aplicadas, não apresentando assim nenhuma CIM clinicamente relevante. Na modulação, o extrato em combinação com os antibióticos testados mostrou efeito sinérgico contra a maioria das bactérias testadas, potencializando o efeito dos antibióticos utilizados. Portanto, este estudo abre caminho para futuras pesquisas que auxiliem a identificar na $S$. spectabilis, moléculas que atuem sinergicamente a antibióticos, tornando-se uma alternativa como adjuvante de antimicrobianos e no combate a infecções bacterianas resistentes às diversas drogas.
\end{abstract}

Palavras-chave: Atividade Antibacteriana. Senna spectabilis. Fitoquímica. Sinergismo.

\begin{abstract}
The objective of the present study was to identify the chemical composition of Senna spectabilis, to investigate the in vitro antimicrobial potential and to analyze its modulating effect of antibiotic activity. Phytochemical assays were performed following the method proposed by Matos (1997), based on visual observation of color change or precipitate formation after the addition of specific reagents. The Minimum Inhibitory Concentration (MIC) and modulation tests were determined by the broth microdilution assay, following the recommendations of the Clinical and Laboratory Standards Institute (CLSI). The strains used were Saphylococcus aureus, Escherichia coli, Pseudomonas aeruginosa and Salmonella enterica. After these analyzes, it was verified the significant presence of the secondary condensed tannin metabolites, alkaloids, steroids and saponins. In the results regarding the evaluation of the antimicrobial action of $S$. spectabilis extract against the tested strains it was observed that there was bacterial growth in all the applied concentrations, thus presenting no clinically relevant MIC. In modulation, the extract in combination with the tested antibiotics showed synergistic effect against most of the tested bacteria, enhancing the effect of the antibiotics used. Therefore, this study paves the way for future research to help identify S. spectabilis molecules that act synergistically to antibiotics, becoming an alternative as an adjunct to antimicrobials and in the fight against bacterial infections resistant to various drugs.
\end{abstract}

Keywords: Antibacterial Activity. Senna spectabilis. Phytochemistry. Synergism.

\section{Introdução}

As plantas medicinais exercem um papel relevante em grande parte dos sistemas tradicionais de medicina, compondo sua base. Mesmo alguns de seus benefícios de cura não possuindo comprovações científicas, os extratos à base de plantas são procurados por conter fontes de compostos bioativos. Entretanto, o seu uso contínuo como tratamento para doenças requer uma análise científica para essas afirmações terapêuticas (NKANTCHOUA et al., 2018).

$\mathrm{O}$ aumento significativo de registros científicos, assim como os relatórios etnofarmacológicos a respeito das plantas brasileiras são um indício de que esta grande riqueza vegetal está distante de ser totalmente elucidada, possuindo um vasto potencial na elaboração de fármacos inovadores (GIULIETTI et al., 2005; RICARDO et al., 2018).

Muitos produtos de origem natural apresentam grande eficácia no combate as infecções microbianas, o que explica as diversas drogas provenientes desses produtos que aumentam cada vez mais, graças a tais benefícios que os mesmos apresentam. Nesse aspecto há um crescente número de pesquisas em desenvolvimento com a finalidade de averiguar novos fármacos com atividade antimicrobiana ou até mesmo moléculas que consigam modular a ação de antibióticos (FERNEBRO et al., 2001; COUTINHO et al., 2008a; VERAS et al., 2013; PONTES et al., 2018).

Como exemplo dessa vasta biodiversidade, a Senna spectabilis ( $\sin$. Cassia spectabilis) é um arbusto leguminoso, que pertence à família Fabaceae (Leguminosae), sendo comumente usada na região do Nordeste brasileiro, na qual 
é conhecida como "canafístula" ou "cassia" (SANTOS et al., 2013).

Esta planta é reconhecida por seu uso ornamental em áreas com climas tropicais e subtropicais. Levando em conta o seu largo valor terapêutico na medicina popular, esta planta tem grande relevância científica (JOTHY et al., 2012). É encontrada no território brasileiro, principalmente na região Nordeste e apresenta, em sua maioria, alcaloides piperidínicos como componentes predominantes, em que são atribuídos a estes metabólitos propriedades tóxicas e farmacológicas de particular interesse, evidenciadas por meio de ensaios com extratos e com substâncias extraídas dessa espécie (VIEGAS JR et al. 2006).

Estudos farmacológicos mostram que a Senna spectabilis possui um amplo espectro de propriedades, alguns exemplos são as atividades antinociceptivas, propriedades analgésicas, anti-inflamatórias, atividades antioxidantes e, também, a atividade antimicrobiana (CALIXTO et al., 2012).

O presente estudo teve como objetivo a definição da composição fitoquímica do extrato hidroalcóolico das folhas de Senna spectabilis, pesquisar o potencial antimicrobiano in vitro através da determinação da concentração inibitória mínima, além de analisar seu efeito modulador frente aos antibióticos.

\section{Material e Métodos}

\subsection{Obtenção do material vegetal}

A coleta das folhas de Senna spectabilis (Cassia) foi realizada no Nordeste, na cidade de Altaneira - CE (Lat. -7.0243390; Log. -39.775810), região do Cariri, na qual a planta cresceu em condições naturais. A coleta ocorreu na época de chuva decorrente de sua floração, no primeiro semestre de 2018. A exsicata foi catalogada no Herbário Caririense Dárdano de Andrade Lima (HCDAL), sob o número 13.621.

\subsection{Preparação do extrato}

$\mathrm{O}$ extrato foi obtido seguindo a metodologia de Ferreira et al. (2016), na qual $386 \mathrm{~g}$ das folhas de $S$. spectabilis foram macerados em 2 litros de etanol a $70 \%$ por 72 horas na ausência de luz. O líquido obtido foi filtrado e, posteriormente, concentrado sob pressão reduzida até a evaporação do álcool, utilizando para tal um rotaevaporador a vácuo (Quimis, São Paulo). O restante do líquido foi levado ao banho-maria a $50{ }^{\circ} \mathrm{C}$ para a evaporação total do solvente, o peso do extrato hidroalcoólico final obtido foi $24,87 \mathrm{~g}$, resultando em um rendimento de $6,4 \%$, sendo e conservado em seguida ao abrigo da luz, e armazenado em geladeira a $4^{\circ} \mathrm{C}$.

\subsection{Micro-organismos}

Foram utilizadas para a análise de atividade microbiana, cepas padrão de bactérias Gram-positivas (Staphylococcus aureus ATCC 6538) e Gram-negativas (Escherichia coli ATCC 25922, Pseudomonas aeruginosa ATCC 9027,
Salmonella enterica ATCC 14028), cedidas pelo laboratório de microbiologia da Faculdade de Medicina Estácio de Juazeiro do Norte.

\subsection{Antimicrobianos}

Os antibióticos utilizados no experimento foram os aminoglicosídeos (amicacina e gentamicina), as lincosamidas (clindamicina), cefalosporinas (cefalexina) e azitromicina antibiótico pertencente a classe azalídeos. As drogas antimicrobianas foram diluídas em água destilada estéril, o resultado final foi de $1024 \mu \mathrm{g} / \mathrm{mL}$ com base nos parâmetros propostos pela Clinical and Laboratory Standards Institute (MELVIN; WEINSTEIN, 2012).

\subsection{Prospecção fitoquímica}

Os ensaios fitoquímicos do extrato de Senna spectabilis foram efetuados seguindo a metodologia proposta por Matos (1997), baseando-se na observação visual da alteração da cor, formando ou não um precipitado logo após do acréscimo dos reagentes específicos.

\subsection{Determinação da Concentração Inibitória Mínima (CIM)}

A definição da CIM (concentração inibitória mínima) ocorreu por meio de ensaio de microdiluição em caldo, seguindo as recomendações do (MELVIN; WEINSTEIN, 2012). Em placas de microdiluição com 96 poços foram distribuídos $100 \mu \mathrm{L}$ do caldo BHI (brain heart infusion) a 3,7\% em todos os orifícios, posteriormente, distribuíram-se $100 \mu \mathrm{L}$ do extrato nos poços da primeira coluna, com diluições na sequência de $1: 1$. No poço final de cada linha foram desprezados os $100 \mu \mathrm{L}$ restantes. As concentrações iniciais e finais do extrato foram de $1024-0,5 \mu \mathrm{g} / \mathrm{mL}$. Introduzidos $100 \mu \mathrm{L}$ de cada linhagem foram empregadas nos respectivos inóculos, suspensas em $900 \mu \mathrm{L}$ de água estéril, apresentando uma concentração de $10^{5}$ UFC (Unidades Formadoras de Colônias) $/ \mathrm{mL}$, seguindo a escala de McFarland (NCCLS, 2008).

Os antibióticos amicacina, clindamicina, gentamicina, azitromicina e cefalexina foram utilizados como controle, realizando da mesma forma do extrato, cujas concentrações iniciais e finais foram de $1024 \mu \mathrm{g} / \mathrm{mL}$ a $0,5 \mu \mathrm{g} / \mathrm{mL}$. Registraram-se as CIMs como as menores concentrações capazes de inibir o crescimento microbiano. Os testes foram feitos em triplicata e ocorrendo a incubação das placas na estufa por 24 horas a temperatura de $36^{\circ} \mathrm{C}$.

\subsection{Determinação da atividade Moduladora}

Para verificar se o extrato modificaria a ação dos antibióticos frente aos micro-organismos testados, utilizou-se o método proposto por Coutinho et al. (2008b), no qual a solução do extrato foi testada em concentração subinibitória (MIC/8). Distribuiu-se em eppendorfs de $2 \mathrm{~mL}$ (para modulação) uma solução contendo BHI $10 \%$, inóculo e o extrato modulado 
(MIC/8). Para a coluna controle (do antibiótico) eppendorfs foram preenchidos com BHI e inóculo. Houve distribuição das soluções dos eppendorfs em colunas nas quais cada poço recebeu $100 \mu \mathrm{L}$ da solução.

Toda a distribuição foi feita no sentido alfabético da placa. Em seguida, $100 \mu \mathrm{L}$ do antibiótico na concentração de $1024 \mu 1 / \mathrm{mL}$ foram misturados ao primeiro poço, ocorrendo a microdiluição em série, em uma proporção de 1:1 até a penúltima cavidade. As concentrações de antimicrobianos variaram gradualmente de 1024 a $1 \mu \mathrm{g} / \mathrm{mL}$. Os testes foram realizados em triplicata e as placas foram incubadas a $36{ }^{\circ} \mathrm{C}$ por $24 \mathrm{~h}$. O efeito sinérgico foi observado a partir da redução da CIM dos antibióticos quando combinados com o extrato utilizado como modulador.

\section{Resultados e Discussão}

Por meio da prospecção fitoquímica do extrato hidroalcoólico das folhas de Senna spectabilis, constatou-se a existência considerável dos metabólitos secundários taninos condensados (coloração verde), alcaloides (aparecimento de precipitado), esteroides (coloração azul seguida de verde permanente) e saponinas (presença de espuma persistente e abundante), ilustrados no Quadro 1, eles manifestam diversificadas atividades biológicas.

Quadro 1 - Composição química do extrato hidroalcoólico das folhas de Senna spectabilis

\begin{tabular}{|c|c|c|c|c|c|c|c|c|c|c|c|c|c|c|c|}
\hline \multicolumn{16}{|c|}{ Metabólitos } \\
\hline \multirow{2}{*}{ EHSS } & 1 & 2 & 3 & 4 & 5 & 6 & 7 & 8 & 9 & 10 & 11 & 12 & 13 & 14 & 15 \\
\hline & - & - & + & - & - & - & - & - & - & - & - & + & - & + & + \\
\hline
\end{tabular}

1 - Fenóis; 2 - Taninos hidrolisáveis; 3 - Taninos condensados; 4 - Antocianinas; 5 - Antocianidinas; 6 - Flavonas; 7 - Flavonóis; 8 - Xantonas; 9 - Chalconas; 10 - Auronas; 11 - Flavonóis; 12 - Esteróides; 13 - Triterpenóides; 14 - saponinas; 15 - Alcalóides; (+) presença; (-) ausência; EHSS - Extrato Hidroalcoólico da Senna spectabilis. / 1 - Phenols; 2 - Hydrolysable tannins; 3 - Condensed tannins; 4 - Anthocyanins; 5 - Anthocyanidins; 6 - Flavones; 7 - Flavonols; 8-Xanthones; 9 - Chalconas; 10 - Auronas; 11 - Flavonols; 12 - Steroids; 13 - Triterpenoids; 14 -saponins; 15 - Alkaloids; $(+)$ presence; (-) absence; EHSS - Hydroalcoholic Extract from Senna spectabilis.

Fonte: Dados da pesquisa.

Segundo pesquisas de Monteiro, Albuquerque e Araújo (2005), os taninos possuem ações importantes contra certos micro-organismos, e atuam também como agentes carcinogênicos e causadores de toxicidade hepática. Podem ter também algumas bioatividades relevantes como a ação anti-inflamatória, cicatrizante e antiviral.

Os esteróides estão ligados ao desenvolvimento e controle do sistema reprodutor humano, atuando como cardiotônicos, precursores de vitamina $\mathrm{D}$, anticoncepcionais orais, antiinflamatórios e, ainda, como agentes anabolizantes. São atribuídas as saponinas a atividade hemolítica, molusquicida, antifúngica, antibacteriana, antiparasitária, citotóxica e antitumoral, antiviral entre outras. Os alcalóides possuem atividades farmacológicas bastante conhecidas, como a morfina, princípio ativo de importante anestésico atual e a tubocurarina um relaxante muscular (ROBBERS et al., 1997; SPARG et al., 2004; CUNHA; FILHO, 2009; BESSA et al., 2013).

Silva et al. (2010) realizaram um estudo sobre a $S$. spectabilis no qual relataram um potencial citotóxico seletivo dos alcalóides piperidínicos espectalina, espectalinina e carnavalina dessa espécie, encontrados nos constituintes dos extratos etanólicos das folhas, frutos e ramos.

Estudos fitoquímicos apontam o isolamento de mais de 350 compostos provenientes do gênero Senna, sendo 40 metabólitos secundários da Senna spectabilis, tais como: alcalóides piperidínicos, triterpenos pentacíclicos, esteróides, pironas, antraquinonas, flavonoides, entre outros, várias ações estão ligadas aos alcalóides de piperidina, estes constituem os principais componentes de folhas, flores e frutos verdes

\section{(SELEGATO et al., 2017).}

Sessenta e oito atividades biofarmacológicas comprovadas foram encontradas nas diversas espécies do gênero Senna, com destaque para as atividades antioxidante e antitumoral, o que pode ser justificado pela presença de compostos fenólicos como antraquinonas e flavonoides nestas plantas. Esta apresenta ainda propriedades anticolinesterásicas, analgésica, antidiabética, antifúngica, anti-inflamatória, antiparasitária, antimicrobiana, antipirética, antiulcera, bactericida e propriedades distintas como abortiva, antidepressiva, antilipidêmica e antiviral (MACEDO; ALAN; SILVA, 2016).

Mesmo apresentando metabólicos secundários em sua composição, o extrato não demostrou atividade antimicrobiana significativa frente as cepas testadas, dispondo de uma CIM com valor $\geq 1024 \mu \mathrm{g} / \mathrm{mL}$.

O Quadro 2 descreve os resultados referentes à avaliação da atividade antimicrobiana do extrato contra as cepas bacterianas de E. coli, S. aureus, P. aeruginosa e S. entérica, em que foi possível observar o crescimento das bactérias em todas as concentrações aplicadas.

Quadro 2 - Determinação da Concentração Inibitória Mínima (CIM) in vitro do extrato hidroalcóolico das folhas de Senna spectabilis

\begin{tabular}{|l|c|}
\hline \multicolumn{1}{|c|}{ Micro-organismos } & CIM $(\mu \mathrm{g} / \mathrm{mL})$ \\
\hline Escherichia coli ATCC $\mathbf{2 5 9 2 2}$ & $\geq 1024$ \\
\hline Staphylococcus aureus ATCC $\mathbf{6 5 3 8}$ & $\geq 1024$ \\
\hline Pseudomonas aeruginosa ATCC 9027 & $\geq 1024$ \\
\hline Salmonella enterica ATCC 14028 & $\geq 1024$ \\
\hline
\end{tabular}

Fonte: Dados da pesquisa. 
Aligiannis et al. (2001) destaca que inibidores fortes têm uma classificação de CIM de até $500 \mu \mathrm{g} / \mathrm{mL}$, inibidores intermediários têm uma CIM entre 600 e $1500 \mu \mathrm{g} / \mathrm{mL}$, CIM acima de $1600 \mu \mathrm{g} / \mathrm{mL}$ classificam-se como inibidores fracos.

Em um estudo sobre a atividade antibacteriana de extratos vegetais e entre esses a Senna spectabilis frente a três isolados bacterianos, que mostraram que os melhores efeitos apresentados da S. spectabilis foram os extratos hidroetanólico e acetato de etila, contra S. aureus, ambos com CIM de 250 $\mu \mathrm{g} / \mathrm{mL}$ (ARANTES et al., 2016).

Em ensaios testando a atividade antimicrobiana do extrato das folhas de Senna spectabilis, empregando solventes com polaridades diversificadas, constataram que os extratos metanólicos e acetona tiveram os melhores resultados de inibição frente às bactérias e fungos, em comparação com os extratos de baixa polaridade, como hexano, diclorometano e acetato de etila (KRISHNAN et al., 2010)

Costa et al. (2015) realizaram uma pesquisa contendo diferentes extratos de S. Spectabilis, mostrando que o extrato de suas flores teve atividade contra os fungos Candida parapsilosis e Candida tropicalis, apresentando uma CIM de $62,5 \mu \mathrm{g} / \mathrm{mL}$ e $15,6 \mu \mathrm{g} / \mathrm{mL}$ respectivamente, enquanto que os extratos das folhas e galhos não tiveram a mesma efetividade, salientando que constituintes químicos da mesma planta se diferem dependendo de quais partes são usadas no estudo.

Algumas plantas do gênero Senna apresentaram atividade antimicrobiana em estudos realizados, como no caso da Senna obtusifolia, seus extratos etanólicos do caule e da folha apresentram atividade antibacteriana frente a Staphylococcus aureus na concentração de $50 \mathrm{mg} / \mathrm{mL}$. Nenhum dos extratos conseguiu inibir as bactérias da espécie de Escherichia coli nas concentrações estudadas (SANTOS, 2013).

Já a Senna occidentalis mostrou uma ação antimicrobiana maior, seu extrato hidroalcoólico de sementes inibiu o crescimento de $S$. aureus (47\%), E. coli (35\%) e P. aeruginosa (78\%). O extrato hidroalcoólico de partes aéreas apresentou atividade contra $S$. aureus (52\%) e P. aeruginosa (59\%), mas a $E$. coli não foi inibida por este extrato (LOMBARDO et al., 2015).

Conforme o Quadro 3, entre os antibióticos analisados, a Gentamicina e Amicacina tiveram melhores resultados frentes às bactérias analisadas, sendo eficazes na inibição da população microbiana das quatro cepas testadas, com CIM's baixas. A Clindamicina teve o resultado inferior comparado aos demais antibióticos, pois este conseguiu inibir o crescimento bacteriano apenas do $S$. aureus, com uma CIM de $\leq 32 \mu \mathrm{g} / \mathrm{mL}$. O antibiótico Azitromicina conseguiu inibir o crescimento das bactérias Pseudomonas aeruginosa $\leq 512 \mu \mathrm{g} /$ $\mathrm{mL}$, Escherichia coli $\leq 426,66 \mu \mathrm{g} / \mathrm{mL}$ e Salmonella enterica $\leq 128 \mu \mathrm{g} / \mathrm{mL}$. A Cefalexina também inibiu o crescimento das bactérias Escherichia coli $\geq 341,33 \mu \mathrm{g} / \mathrm{mL}$ e Salmonella enterica $\leq 0,5 \mu \mathrm{g} / \mathrm{mL}$.

Quadro 3 - CIM dos controles antibióticos; E. coli (Escherichia coli); S. aureus (Staphylococcus aureus); S. enterica (Salmonella enterica); $P$. aeruginosa (Pseudomonas aeruginosa)

\begin{tabular}{|l|c|c|c|c|}
\hline \multirow{4}{*}{ Antibióticos } & \multicolumn{4}{|c|}{ Micro-organismos e CIM $(\mu \mathrm{g} / \mathbf{m L})$} \\
\cline { 2 - 5 } & $\begin{array}{c}\text { E. coli } \\
\text { ATCC } \\
\mathbf{2 5 9 2 2}\end{array}$ & $\begin{array}{c}\text { S. aureus } \\
\text { ATCC } \\
\mathbf{6 5 3 8}\end{array}$ & $\begin{array}{c}\text { S. enterica } \\
\text { ATCC } \\
\mathbf{1 4 0 2 8}\end{array}$ & $\begin{array}{c}P . \\
\text { aeruginosa } \\
\text { ATCC } \\
\mathbf{9 0 2 7}\end{array}$ \\
\hline Amicacina & $\leq 32$ & $\leq 32$ & $\leq 8$ & $\leq 64$ \\
\hline Clindamicina & $\geq 1024$ & $\leq 32$ & $\geq 1024$ & $\geq 1024$ \\
\hline Gentamicina & $\leq 0,5$ & $\leq 8$ & $\leq 8$ & $\leq 16$ \\
\hline Azitromicina & $\leq 426$ & $\geq 1024$ & $\geq 128$ & $\leq 512$ \\
\hline Cefalexina & $\leq 341$ & $\geq 1024$ & $\leq 0,5$ & $\geq 1024$ \\
\hline
\end{tabular}

Fonte: Dados da pesquisa.

O Quadro 4 mostra a potencialização do efeito em combinação com os antibióticos. $\mathrm{Na}$ atividade moduladora contra as bactérias, foi observado resultado sinérgico com os antibióticos Amicanica, Azitromicina e Cefalexina.

Quadro 4 - A (CIM dos antibióticos isolada em $\mu \mathrm{g} / \mathrm{mL}$ ); +EHSS (CIM do efeito modulador do extrato junto ao antibiótico em $\mu \mathrm{g}$ / $\mathrm{mL}$ ); E. coli (Escherichia coli); S. aureus (Staphylococcus aureus); S. enterica (Salmonella enterica); P. aeruginosa (seudomonas aeruginosa); EHSS - Extrato Hidroalcoólico da Senna spectabilis

\begin{tabular}{|l|c|c|c|c|c|c|c|c|}
\hline & \multicolumn{2}{|c|}{$\begin{array}{c}\text { E. coli } \\
\text { ATCC 25922 }\end{array}$} & \multicolumn{2}{c|}{$\begin{array}{c}\text { S. aureus } \\
\text { ATCC 6538 }\end{array}$} & \multicolumn{2}{c|}{$\begin{array}{c}\text { S. enterica } \\
\text { ATCC 14028 }\end{array}$} & \multicolumn{2}{c|}{$\begin{array}{c}\text {. aeruginosa } \\
\text { ATCC 9027 }\end{array}$} \\
\hline & A & + +EHSS & A & + EHSS & A & +EHSS & A & +EHSS \\
\hline Amicacina & $\leq 32$ & $\leq 1$ & $\leq 32$ & $\leq 32$ & $\leq 8$ & $\leq 1$ & $\leq 64$ & $\leq 1$ \\
\hline Clindamicina & $\geq 1024$ & $\geq 1024$ & $\leq 32$ & $\geq 1024$ & $\geq 1024$ & $\geq 1024$ & $\geq 1024$ & $\geq 1024$ \\
\hline Gentamicina & $\leq 0,5$ & $\leq 1$ & $\leq 8$ & $\geq 1024$ & $\leq 8$ & $\geq 1024$ & $\leq 16$ & $\leq 682$ \\
\hline Azitromicina & $\leq 426$ & $\leq 1$ & $\geq 1024$ & $\leq 181$ & $\leq 128$ & $\leq 1$ & $\leq 512$ & $\leq 1$ \\
\hline Cefalexina & $\leq 341$ & $\leq 1$ & $\geq 1024$ & $\geq 1024$ & $\leq 0,5$ & $\geq 1024$ & $\geq 1024$ & $\leq 1$ \\
\hline
\end{tabular}

Fonte: Dados da pesquisa.

O extrato combinado à Amicacina mostrou efeito sinérgico contra 3 das 4 bactérias testadas, potencializando o antibiótico utilizado frente E. coli, $P$. aeruginosa e $S$. enterica, a CIM para o micro-organismo $S$. aureus permaneceu a mesma. $\mathrm{Na}$ combinação do extrato com a Clindamicina observou que não houve atividade moduladora contra as bactérias testadas. Quando utilizado junto a Gentamicina, observou nos resultados obtidos um aumento na CIM em todas as bactérias (E. coli, P. aeruginosa, S. aureus e $S$. entérica), verificando que o extrato antagonizou o efeito deste antibiótico.

A associação do extrato com Azitromicina mostrou efeito sinérgico contra todas bactérias testadas, reduzindo 
significativamente a CIM desse antibiótico quando utilizada frente $E$. coli, $S$. aureus, $P$. aeruginosa e $S$. entérica. O extrato associado a Cefalexina apresentou sinergismo quando testado contra E. coli, $P$. aeruginosa, reduzindo consideravelmente a CIM, mas no teste frente a $S$. entérica houve um grande aumento da sua CIM, observando que o extrato antagonizou seu efeito. Nos ensaios com o micro-organismo S. aureus a CIM permaneceu a mesma, não tendo sinergismo ou antagonismo.

Embora a atividade antimicrobiana do extrato testado neste estudo não tenha sido considerada significativa, quando associada aos antibióticos, tal extrato proporcionou a redução da CIM sobre as cepas.

Várias plantas possuem metabólitos secundários que podem ser boas substâncias para a terapia combinada, seus mecanismos de ação podem ter relação com o aumento da permeabilidade da membrana celular externa, através da inibição das modificações do sítio ativo do fármaco, inibição de bombas de efluxo ou evitando a inativação do antibiótico por meio da degradação ou modificação enzimática (HEGDE et al. 2004; ROCCARO et al., 2004; WRIGHT, 2005; HEMAISWARYA et al., 2008).

Compostos fenólicos como taninos podem ser tóxicos para micro-organismos por causa da complexação com outras moléculas e, portanto, na inativação destas como de enzimas e proteínas transportadoras da parede celular (COWAN, 1999; KIM et al., 2004; FABRI; COSTA, 2012).

Figueredo et al. (2013) destaca o efeito relevante da ação moduladora dos metabólitos secundários de plantas medicinais sobre os aminoglicosídeos, podendo se tornar uma alternativa para a diminuição das reações adversas causadas por esses antimicrobianos, tendo em vista que a interação planta-antibiótico possui a capacidade de diminuir a CIM destas drogas e, por consequência, reduzir a dose necessária para o uso terapêutico.

\section{Conclusão}

O extrato hidroalcoólico das folhas de Senna spectabilis (EHSS) apresentou em sua triagem fitoquímica os metabólitos taninos condensados, esteroides, saponinas e alcaloides, os quais apresentam atividades biológicas.

Nos testes de CIM, apesar do extrato não ser capaz de inibir o crescimento microbiano, observou-se que na associação houve sinergismo em combinação com as drogas Amicacina, Azitromicina e Cefalexina, apresentando desempenho favorável na modulação da atividade antibiótica, potencializando seus efeitos.

O presente estudo abre caminho para futuras pesquisas que auxiliem a identificar na $S$. spectabilis, moléculas que atuem sinergicamente aos antibióticos, tornando-se uma alternativa no combate a infecções microbianas resistentes às diversas drogas.

\section{Referências}

ALIGIANNIS, N. et al. Composition and antimicrobial activity of the essential oil of two Origanum species. J. Agric. Food Chem., v.49, n.9, p.4168-4170, 2001. doi: 0.1021/jf001494m

ARANTES, V.P. et al. Estudo comparativo da atividade antibacteriana de extratos vegetais de Senna spectabilis, Rosmarinus officinalis e Eugenia uniflora frente à cepa padrão de Pseudomonas aeruginosa ATCC 27853, Staphylococcus aureus ATCC 6538 e Streptococcus pyogenes ATCC 19615. Arq. Cienc. Saúde UNIPAR, v.20, n.3, p.151-158, 2016. doi: 10.25110/ arqsaude.v20i3.2016.5715

BESSA, N.D. et al. Prospecção fitoquímica preliminar de plantas nativas do cerrado de uso popular medicinal pela comunidade rural do assentamento vale verde - Tocantins. Re. Bras. Plant. Med., v.15, n.4, p.692-707, 2013. doi: 10.1590/S151605722013000500010

CALIXTO, J.B. et al. Plant derived alkaloid (-)-cassine induces anti-inflammatory and anti-hyperalgesics effects in both acute and chronic inflammatory and neuropathic pain models. Neuropharm., v.62, n.2, p.967-977, 2012. doi: 10.1016/j. neuropharm.2011.10.002

COUTINHO, H.D. et al. In vitro interference of Momordica charantia and chlorpromazine in the resistance to aminoglycosides. Pharm. Biol., v.47, n.11, p.1056-1059, 2009. doi: $10.3109 / 13880200902991540$

COUTINHO, H.D. et al. Enhancement of the antibiotic activity against a multiresistant Escherichia coli by Mentha arvensis L. and chlorpromazine. Chemoth., v.54, n.4, p.328-330, 2008. doi: $10.1159 / 000151267$

COSTA, G.M. et al. Effect of plant extracts on planktonic growth and biofilm of Staphylococcus aureus and Candida albicans. International J. Curr. Microb. Appl. Scie., v.4, n.6, p.908-917, 2015.

COWAN, M.M. Plant products as antimicrobial agents. C. Microb. Rev., v.12, n.4, p.564-582, 1999. doi: 10.1128 / CMR.12.4.564

CUNHA, E.V.L.; BARBOSA-FILHO, J.M. Alcalóides derivados do núcleo isoquinolínico. In: YUNES, R.A.; CECHINEL FILHO, V. Química de produtos naturais, novos fármacos e a moderna farmacognosia. Itajaí; Santa Catarina: UNIVALI, 2009.

FABRI, R.L.; COSTA, J.A.B.M. Perfil farmacognóstico e avaliação das atividades citotóxica e antibacteriana de Bromelia antiacantha Bertol. Rev. Eletr. Farm., v.9, n.2, p.12, 2012. doi: 10.5216/ref.v9i2.18427

FERNEBRO, J. Fighting bacterial infections: future treatment options. D. Resist. Upd., v.14, n.2, p.125-139, 2011. doi: 10.1016/j.drup.2011.02.001

FERREIRA, J.V.A. et al. Avaliação da atividade antimicrobiana e moduladora do extrato etanólico de Libidibia ferrea (Mart. ex Tul.) L.P. Queiroz. Rev. Cub. Plant. Medic., v.21, p.1, 2016.

FIGUEREDO, F.G. et al. Modulation of the Antibiotic Activity by Extracts from Amburana cearensis A. C. Smith and Anadenanthera macrocarpa (Benth.) Brenan. BioMed Res. Intern., p.1-5, 2013. doi: 10.1155/2013/640682

GIULIETTI, A.M. et al. Biodiversity and conservation of plants in Brazil. Conserv. Biol., v.19, n.3, p.632-639, 2005. doi: 10.1111/j.1523-1739.2005.00704.x

HEGDE V.R. et al. Two new bacterial DNA primase inhibitors from the plant Polygonum cuspidatum. B. Med. Chem. Let., v.14, n.9, p.2275-2277, 2004. doi: 10.1016/j.bmcl.2004.02.006

HEMAISWARYA, S.; KRUTHIVENTI, A.K.; DOBLE, M. 
Synergism between natural products and antibiotics against infectious diseases. Phytomed., v.15, n.8, p.639-652, 2008. doi: 10.1016/j.phymed.2008.06.008

JOTHY, S.L. et al. Cassia spectabilis (DC) Irwin et Barn: a promising traditional herb in health improvement. Molecules, v.17, n.9, p.10292-10305, 2012. doi: 10.3390/molecules 170910292

KIM, H.P. et al. Anti-inflammatory plant flavonoids and cellular action mechanisms. J. Pharm. Sci., v.96, n.3, p.229-245, 2004. doi: 10.1254/jphs.CRJ04003X

KRISHNAN, N. et al. Antimicrobial activity evaluation of Cassia spectabilis leaf extracts. Int. J. Pharm., v.6, n.4, p.510-514, 2010. doi: 10.3923/ijp.2010.510.514

LIMA, R.A. et al. How much do we know about the endangered Atlantic forest? Reviewing nearly 70 years of information on tree community surveys. B. Conserv., v.24, n.9, p.2135-2148, 2015. doi: 10.1007/s10531-015-0953-1

LOMBARDO, M. et al. Evaluation of in vitro biological properties of Senna occidentalis (L.) Link. A. Scient.. He. Sci., v.37, n.1, p.9-13, 2015. doi: 10.4025/actascibiolsci.v37i1.22525

MACEDO, E.M.; ALAN E SILVA, J.G.; SILVA, M.G.V. Quimiodiversidade e propriedades biofarmacológicas de espécies de Senna nativas do Nordeste do Brasil. Rev. Virt. Quím., v.8, n.1, p.169-195, 2016. doi: 10.5935/1984-6835.20160012

MATOS, F.J.A. Introdução à fitoquímica experimental. Fortaleza: UFC Edições, 1997.

MELVIN, P.; WEINSTEIN, M.D. Methods for dilution antimicrobial susceptibility tests for bacteria that grow aerobically. Pennsylvania: Clinic. Laborat. Stand. Inst., 2012.

MONTEIRO, J.M.; ALBUQUERQUE, U.P.; ARAÚJO, E.L. Taninos: uma abordagem da química à ecologia. Quím. Nova, v.28, n.5, p.892, 2005. doi: 10.1590/S0100-40422005000500029.

NCCLS. National Comitee for Clinical Laboratory Standards. Methods for dilution antimicrobial susceptibility tests for bacteria that grow aerobically: Approved standard, 6th ed. NCCLS document M7-A6. Wayne: National Committee for Clinical Laboratory Standards, 2008.

NKANTCHOUA, G.N. et al. Anticonvulsant effects of Senna spectabilis on seizures induced by chemicals and maximal electroshock. J. Ethnopharm., v.212, p.18-28, 2018. doi: 10.1016/j.jep.2017.09.042

PONTES, M. A. et al. Efeito Inibitório De Monoterpenos Frente A Klebsiella Pneumoniae Produtoras de ESBL. Rev. Bras. Ciênc. S., v.22, n.1, p.51-56, 2018. doi: 10.4034/RBCS.2018.22.01.07

RICARDO, L.M. et al. Evidence of traditionality of Brazilian medicinal plants: The case studies of Stryphnodendron adstringens (Mart.) Coville (barbatimão) barks and Copaifera spp.(copaíba) oleoresin in wound healing. J. Ethnopharm., v.219, p.319-336, 2018. doi: 10.1016/j.jep.2018.02.042

ROBBERS, J.E.; SPEEDIE, M.K.; TYLER, V.E. Farmacognosia e farmacobiotecnologia. São Paulo: Editorial Premier, 1997.

ROCCARO, A.S. et al. Epigallocatechin-Gallate Enhances the Activity of Tetracycline in Staphylococci by Inhibiting Its Efflux from Bacterial Cells. Ant. Ag. Chemoth., v.48, n.6, p.1968-1973, 2004. doi: 10.1128/AAC.48.6.1968-1973.2004

SANTOS, A. F. Atividade antibacteriana, antioxidante e toxicidade do extrato etanólico de Senna obtusifolia. Rev. Eletr. Farm., v.10, n.3, p.11-11, 2013. doi: 10.5216/ref.v10i3.26913

SANTOS, M. et al. Determination of the genetic diversity among accessions of Senna spectabilis (canafístula) by using RAPD markers. Gen. Mol. Res., v.12, n.4, p.6149-6155, 2013. doi: 10.4238/2013.December.2.12

SELEGATO, D.M. et al. Update: Biological and chemical aspects of Senna spectabilis. J. Braz. Chem. Soc., v.26, p.415-426, 2017. doi: 10.21577/0103-5053.20160322

SILVA, D.H. et al. Espectalina, Cassina e Análogos Semissintéticos como Potenciais Candidatos a Fármacos para o Tratamento da Doença de Alzheimer. Rev. Virt. Quím., p.38-46, 2010. doi: 10.5935/1984-6835.20100005

SPARG, S. G.; LIGHAT, M. E.; VAN STADEN, J. Biological activities and distribution of plant saponins. J. Ethnopharm., v.94, n.2-3, p. 219-243, 2004. doi: 10.1016/j.jep.2004.05.016

VERAS, H.N. et al. Enhancement of aminoglycosides and b-lactams antibiotic activity by essential oil of Lippia sidoides Cham. and the Thymol. Ar. J. Chem., v.10, p.S2790-S2795, 2017. doi: 10.1590/S0100-40422006000600025

WRIGHT, G. D. Bacterial resistance to antibiotics: enzymatic degradation and modification. Adv. Drug Del. Rev., v.57, n.10, p.1451-1470, 2005. doi: 10.1016/j.addr.2005.04.002 